\title{
Modelos, discursos y perspectivas teóricas vigentes sobre discapacidad y deficiencia
}

\author{
Marisa Andrea Cenacchi
}

\begin{abstract}
Resumen
La discapacidad constituye un campo multidimensional complejo, atravesado por diversos discursos y teorías que han caracterizado a las formas de nombrar y conceptualizar a la alteridad deficiente en el tiempo. Considerando que los discursos construyen los objetos e influyen en los patrones de respuesta que se derivan o se implican en ellos, se presenta necesario deconstruir los conceptos heredados sobre dicho colectivo para habilitar la reflexión crítica sobre la vigencia explícita y/o implícita en los contextos educativos actuales y sus implicancias.

En esta dirección, se presentan los principales modelos históricos y perspectivas teóricas críticas más significativas que abonan el campo. Si bien hay consenso en concebir a la discapacidad como una construcción social, se observan divergencias en la conceptualización de las deficiencias. Aún desde el modelo social, se sostiene una división teórica entre discapacidad y deficiencia que mantiene vigente el modelo médico, el concepto de déficit y su contracara, la normalidad. Por el contrario, diversas perspectivas críticas sustentadas en los estudios culturales, feministas, poscoloniales y teorías posmodernas, proponen suprimir esa división binaria y concebir también a la deficiencia como socialmente construida.
\end{abstract}

Palabras clave: discapacidad, alteridad deficiente, estudios sobre discapacidad, estudios críticos en discapacidad

\begin{abstract}
* Profesora en Educación Musical, Universidad Nacional de Rosario (UNR), Doctoranda en Humanidades y Artes, Mención Ciencias de la Educación (UNR), Coordinadora Académica del Campus Virtual (UNR). e-mail: macenacchi@gmail.com
\end{abstract}


Modelos, discursos y perspectivas teóricas

\section{Current models, discourses and theoretical perspectives on disability and deficiency}

\section{Abstract}

Disability is a complex multidimensional field, crossed by a variety of discourses and theories about labelling and conceptualizing the impaired alterity over the years. Discourses build objects and influence the response patterns that are derived or implied in them. Thus, it is necessary to deconstruct inherited concepts about that collective in order to enable critical reflection on explicit and/or implicit validity in educational contexts and their implications.

That is why, the main historical models and critical theoretical perspectives of the field are presented. While there is an agreement on conceiving disability as a social construction, there are divergences observed in the conceptualization of deficiencies. Even from the point of view of the social model, there is a theoretical division between disability and deficiency that keeps the medical model in effect, the concept of deficit and its opposite, normality. By the other hand, a range of critical perspectives based on cultural, feminist, postcolonial studies and postmodern theories, propose to suppress this binary division and to conceive also deficiency as socially constructed.

Keywords: disability, impaired alterity, disability studies, critical disability studies 


\section{Introducción}

En la actualidad es posible afirmar que la discapacidad no se constituye como un hecho objetivo y natural, sino que es una construcción, atravesada por diferentes teorías y discursos, resultado de consensos y, por lo tanto, artificial e inscripta en un contexto socio-histórico y político situado, con objetivos y necesidades específicas.

Siguiendo a Foucault (2002), los discursos son prácticas que configuran sistemáticamente los objetos de los que hablan, no se refieren a objetos; sino que los construyen y, al hacerlo, ocultan su propia invención. Así, los discursos generan un marco de posibilidad sobre lo que es, sobre lo que puede ser dicho y pensado, pero también sobre quién tiene autoridad para hablar, a quién y cuándo, construyendo tanto la subjetividad como las relaciones de poder (Ball, 1993). Por lo tanto, las convenciones lingüísticas no sólo estructuran los significados asignados al término discapacidad y demás formas de nombrar ${ }^{1}$ a la alteridad deficiente (Skliar, 2002), sino que también influyen en los patrones de respuesta que se derivan o se implican en ellos (Linton, 2006). En esta línea, algunos autores han conceptualizado a la discapacidad como una categoría inestable (Davis, 2006) que dependerá del marco discursivo desde donde se la aborde.

Por este motivo, en el marco de las actuales políticas educativas inclusivas nacionales e internacionales y el impulso que los instrumentos legales otorgan a la recuperación del derecho a la educación del colectivo identificado como personas con discapacidad, resulta imprescindible deconstruir los conceptos y supuestos heredados sobre dicho colectivo, que habilitan, enmarcan y sustentan las prácticas educativas. Según Derrida (1986), la deconstrucción es una estrategia de análisis que permite deshacer sin destruir un sistema de pensamiento hegemónico, para dar lugar a la transformación, al acontecimiento, al imprevisible "por-venir".

\footnotetext{
${ }^{1}$ En el presente trabajo se utilizarán los términos relevantes de cada momento histórico y/o posicionamiento teórico: anormal, deficiente, persona con discapacidad, etc.
} 
Entonces, en el presente artículo se realizará una revisión de los principales discursos, modelos históricos, paradigmas y perspectivas teóricas críticas más significativas que abonan el campo de la discapacidad, para habilitar la reflexión crítica y el análisis de la vigencia explícita y/o implícita en los contextos actuales.

\section{Modelos históricos}

La temática de la discapacidad constituye un campo de abordaje multidimensional complejo. Diversos autores (Aguado Diaz, 1995; De Jong, 1979; Palacios, 2008; Puig de la Bellacasa, 1990), han propuesto paradigmas o modelos para caracterizar las formas de tratamiento diferencial dispensado hacia la alteridad deficiente a lo largo del tiempo. En ellos se incluyen las representaciones y el conjunto de relaciones y valoraciones sociales en los que se inscribe a los sujetos señalados como deficientes.

Inicialmente De Jong (1979) introduce en el campo problemático de la discapacidad la noción de paradigma de Khun, concibiendo que un paradigma define la realidad para un científico, provee el marco donde identifica sus problemas y los resuelve. Cuando una serie de anomalías no pueden ser resueltas por el paradigma dominante se produce una crisis al interior de una comunidad científica, que genera la búsqueda de uno alternativo que aporte soluciones. De Jong propone la transposición de estos conceptos al campo de la discapacidad, señalando la necesidad de abandonar el paradigma de la rehabilitación y adoptar un nuevo paradigma, el de vida independiente, para dar respuestas a problemas no contemplados en el anterior.

Una diferente organización propone Aguado Díaz (1995) en su abordaje histórico de las deficiencias. El autor elige como criterio de demarcación temporal las llamadas tres revoluciones en salud mental, donde los cambios que determinan cada punto de inflexión no suponen el quiebre y la anulación de las concepciones vigentes. Por el contrario, la novedad opera en simultaneidad y conflicto con las prácticas y conceptos anteriores coexistiendo conjuntamente. 
Así, distingue un primer período que va desde la prehistoria hasta a finales del siglo XVI d.C. y principios del XVII, momento en el que ubica la primera revolución en salud mental, inscripta dentro del proceso de laicización del individuo impulsado por el humanismo renacentista. En esta revolución se marca el paso del pecado a la enfermedad, de un modelo divino, mágico y/o naturalista a un modelo organicista y biologicista, cuyo símbolo es la creación de instituciones manicomiales. La segunda revolución la ubica a finales del XIX con surgimiento de las ciencias sociales, la psiquiatría, el psicoanálisis, la psicología clínica, entre otras, donde se observa el paso de un modelo organicista a otro intrapsíquico y mentalista. Finalmente, establece una tercera revolución en los años sesenta determinada por la inflexión hacia concepciones donde se acentúan los determinantes psicosociales y socioambientales y, como correlato, la intervención comunitaria.

Asimismo, distingue dos enfoques que coexisten históricamente en la forma de concebir a las deficiencias: el enfoque pasivo, que las considera fruto de causas ajenas al hombre y, por tanto, como situación incontrolada e inmodificable que da lugar a actitudes de rechazo, segregación, marginación, etc. En contraposición, el enfoque activo aborda la deficiencia como producto de causas naturales, biológicas y/o ambientales y, por tanto, constituyen una situación modificable. En esta dirección se desprenden prácticas tales como la prevención, el tratamiento, la integración, etc.

Por su parte, la propuesta de Puig de la Bellacasa (1990) organiza la evolución histórica de los modelos de atención con el siguiente ordenamiento: el modelo tradicional, el paradigma de la rehabilitación y el paradigma de la autonomía personal o vida independiente.

Finalmente, Palacios (2008), retoma lo anterior y propone la siguiente organización desarrollada a continuación: Modelo Tradicional o de Prescindencia con sus variantes Eugenésico y de Marginación; Modelo Médico o Rehabilitador, y por último, el denominado Modelo Social de la Discapacidad. 
Modelos, discursos y perspectivas teóricas

\section{Modelo Tradicional o de Prescindencia}

Desde esta perspectiva predomina el enfoque pasivo ${ }^{2}$ y las personas con discapacidad se consideran víctimas de castigos divinos o del mal, causas ajenas a su voluntad que, por lo tanto, no pueden ser evitadas. En este marco, se concibe que los sujetos de este colectivo no aportan a la sociedad, son innecesarios e incluso resultan una carga por lo que se decide prescindir de ellos.

En el submodelo Eugenésico se prescinde de la alteridad deficiente mediante la aplicación de políticas eugenésicas, es decir, con su eliminación. El infanticidio fue una práctica muy común en la antigüedad basada en la consideración maligna del origen de las deficiencias congénitas. Según Aguado Díaz (1995), en la antigua India, los niños deformes eran arrojados al Ganges, en Esparta se despeñaban por el monte Taigeto, mientras que, en Atenas, se les dejaba abandonados a la puerta de un templo por si alguien los adoptaba. Se observa, no obstante, diferente trato para los sujetos con deficiencia adquirida en situación bélica, quienes en Atenas recibían pensiones mientras que en Roma compartían el reparto de alimentos y territorios.

Por su parte, en el submodelo de la Marginación que caracterizó a la Edad Media, devenido del cristianismo, se prescinde de los sujetos deficientes situándolos en un espacio separado. Ahora, según Palacios (2008), las personas con discapacidad pasan a ocupar un lugar en la sociedad, el de los marginados, generalmente mendigos quienes recibían la caridad de los ricos y el asilo de la Iglesia. Incluso se llega a la formalización de las limosnas y a la profesionalización del estatus del mendigo, que tiende a perpetuar su condición y fijarla, manteniendo la posición de inferioridad de forma permanente.

El asistencialismo, la caridad, la beneficencia son rasgos típicos que derivan de estas concepciones, donde siempre se establece

\footnotetext{
${ }^{2}$ El enfoque activo observa en menor medida: en una prótesis que data del 2.300 a.C aprox. en Kazajistán y en Egipto, en grabados y esculturas de ortesis del 2.800 a.C y en la mano artificial más antigua fechada en el 2.000 a.C. (Aguado Díaz, 1995)
} 
una relación asimétrica entre los sujetos signada por la marginación, segregación, estigmatización, subestimación y dependencia de las personas con discapacidad, quienes no son considerados como sujetos de iguales derechos que el resto de los ciudadanos. Según Puig de la Bellacasa (1990), los esquemas asistenciales que se iniciaron en la Edad Media desde la beneficencia y caridad de la iglesia, seguirán con la asistencia como aspecto de orden público (siglos XVI y XVII), como derecho legal (siglos XIX y XX) y como seguridad social y políticas compensatorias en la actualidad.

\section{Modelo Médico o Rehabilitador}

El segundo modelo, llamado Médico o Rehabilitador, considera a la discapacidad como una problemática individual o "tragedia personal", consecuencia directa de limitaciones funcionales procedentes de un déficit: deficiencia física, mental o sensorial del sujeto, en relación a un estado normal, definido según parámetros biológicos, psicológicos y médicos. Sus causas ya no son religiosas sino científicas, por lo que debe ser correspondientemente diagnosticada, tratada y certificada. Desde esta perspectiva se persigue, mediante el esfuerzo de la persona con discapacidad en asistencia de la ciencia médica, su rehabilitación y normalización, es decir, la modificación de su estado en pos de poder equipararse en la medida de lo posible al resto de la sociedad.

El sujeto se convierte así en un objeto de estudio e intervención de la Medicina. El médico será el experto técnico competente, capaz de cuidar y prescribir los procedimientos idóneos para su recuperación. Entre ellos pueden incluirse desde cirugías complejas, hasta tratamientos farmacológicos, fisiológicos, fonoaudiológicos, de estimulación sensorial, entre otros. Los avances médicos acompañan el despliegue de este modelo y representan una mejora en la vida de quienes nacen con problemáticas congénitas, así como también en el desarrollo de tratamientos tanto preventivos como rehabilitadores.

La categoría estática y definitiva de anormalidad se modifica y los sujetos ya no son considerados inútiles para la sociedad, sino 
que éstos pueden aportar en la medida en que se "recuperen" o se "adapten" mediante tratamiento médico, terapéutico y/o educativo.

Según Aguado Díaz (1995) uno de los hitos documentados más antiguos sobre la posibilidad de modificación de una deficiencia data de mediados del siglo XVI, cuando el monje Pedro Ponce de León desafió lo establecido por Hipócrates y Aristóteles y enseñó a hablar a un "sordomudo". Iniciativas similares se continuaron en la Modernidad, sin embargo, el modelo comienza a desarrollarse con fuerza a fines del siglo XIX y se consolida iniciando el siglo XX, luego de la Primera Guerra Mundial, a raíz de los heridos que esta dejó junto con los accidentados laborales de la incipiente sociedad industrial.

\section{Modelo Social de la Discapacidad}

Finalmente, el tercer modelo denominado Social, también conocido como de la Autonomía Personal o Vida Independiente, es aquel que considera que las causas que originan a la discapacidad son sociales. Desde esta perspectiva, el problema no es la diferencia biofísica o deficiencia, sino el entorno, que no ofrece oportunidades equitativas para que cada integrante de la sociedad, cualesquiera sean sus condiciones físicas, sensoriales o mentales, pueda circular, participar, informarse, comunicarse y desarrollarse. El énfasis se corre a la "rehabilitación" de la sociedad en su conjunto, la cual no ha tenido en cuenta sus particularidades y requerimientos en la mayoría de diseños, entornos, productos, construcciones, entre otras.

El planteamiento de base, de raíz materialista, radica en que la discapacidad es una "construcción social", impuesta por el grupo hegemónico, y, por lo tanto, las personas con discapacidad constituyen una minoría, una clase social oprimida.

Desde esta perspectiva se concibe que las personas con discapacidad pueden aportar en igual medida que los demás y se apunta a que puedan alcanzar su autonomía, a tal fin, se enfocan en detectar y eliminar las barreras que los discapacitan e impiden u obstaculizan su participación con condiciones equitativas. 
Sus antecedentes se sitúan en las décadas del sesenta y setenta en Estados Unidos y Gran Bretaña, en el marco de los movimientos por los derechos humanos. Ha sido el propio colectivo el que comenzó a pensar y plantear una teoría sobre sí mismos, contrapuesta al modelo biomédico dominante.

En 1975 se forma el grupo Union of the Physically Impaired Against Segregation (Unión de Personas con Insuficiencias Físicas contra la Discriminación - UPIAS) para compartir experiencias y comenzar a organizar sus luchas personales de modo conjunto (UPIAS, 1975).

Posteriormente, Oliver plantea en 1983 el "Modelo Social de la Discapacidad" y en 1987 desarrolla su explicación materialista de la producción de la discapacidad como condición medicalizada e individualizada dentro de las relaciones sociales de la producción capitalista (Oliver, 1998). El movimiento cobra fuerza con la aparición de revistas y publicaciones específicas que desarrollan diferentes aspectos del modelo, con una fuerte producción teórica en la década del noventa. Así, se abre el campo de los llamados "Estudios sobre discapacidad" ("Disability Studies", en adelante DS) caracterizados por una severa crítica al modelo médico.

Según Barton (2009), es un modelo sociopolítico que cumple diversas funciones. En primer lugar, proporciona un marco para que las personas con discapacidad puedan describir sus experiencias que reflejan especialmente actos de discriminación, exclusión y desigualdad, y puedan compartirlas, debatirlas, analizarlas y cuestionarlas. En segundo lugar, ofrece una explicación de la discapacidad en el marco de las relaciones socioeconómicas. En tercer lugar, favorece el apoyo y compromiso colectivo. Finalmente, permite la construcción de una alternativa de vida y una posibilidad de proyectar una imagen positiva de la discapacidad hacia la sociedad que no posee discapacidad.

\section{Modelo de la diversidad}

Iniciado el siglo XXI surge en España, en el marco de las DS, el llamado "Modelo de la Diversidad" que se centra en el concepto 
de dignidad, inherente a los seres humanos y no vinculado a la capacidad.

Desde esta perspectiva se impulsa un cambio en la terminología por considerar que podría influir sobre la situación de los sujetos, dado que lenguaje y pensamiento están ligados entre sí. En este sentido, el termino diversidad funcional presentado constituye

la primera denominación de la historia en la que no se da un carácter negativo ni médico a la visión de una realidad humana, y se pone énfasis en su diferencia o diversidad, valores que enriquecen al mundo en que vivimos. (Romanach \& Lobato, 2005, p. 8)

Los autores proponen utilizar el término "personas con diversidad funcional visual" para identificar determinados colectivos que poseen barreras comunes. Además, algunos autores adoptan dicho término en sustitución de "deficiencia" mientras que se sostiene el de "discapacidad" para referirse a la sociedad y su opresión sobre un colectivo determinado (Palacios, 2008).

No obstante, se observa que, en muchas oportunidades, el termino diversidad funcional se utiliza para la identificación de un colectivo, distinguiendo personas con diversidad funcional de las personas sin diversidad funcional, lo que deriva nuevamente en una categorización que limita las posibilidades de significación del término. Precisamente se estima que su uso puede acompañar a un cambio en el abordaje de la problemática y resultar constructivo en la práctica en su calidad de característica intrínseca a todas las personas, y no solo de determinados colectivos. En efecto, el término no hace referencia a un déficit, una falta en relación con una supuesta normalidad sino a una particularidad extensiva a todos los seres humanos, ya que, en mayor o menor grado, las diferencias culturales, etarias, preferencias cognitivas, gustos, situaciones circunstanciales, etc., hacen que sea posible pensar de manera extrema, en tantas diversidades funcionales como seres humanos. Así, el término permite un uso amplio y abierto, al referir a un rasgo no específico de determinados colectivos ni relacionado unívocamente con una "deficiencia". 


\section{Perspectivas críticas}

A partir de investigaciones que focalizan la experiencia de las personas con discapacidad, vinculadas especialmente con el abordaje de la identidad, la corporalidad y la agencia, se conforma un nuevo campo, el los "Estudios Críticos en Discapacidad" ("Critical Disability Studies", en adelante CDS) (Meekosha, 2011; Meekosha \& Shuttleworth, 2009, Shildrick, 2012, Tremain, 2001, Vehmas \& Watson, 2014). Según Goodley (2013), el campo de los CDS se constituye en una plataforma desde donde pensar, actuar, resistir, relacionar, comunicar y vincularse con otros en contra de las formas hibridadas de opresión y discriminación, más allá de la discapacidad.

El punto común de estos trabajos, es su distancia con el modelo social tradicional promovido desde Reino Unido. Esta perspectiva considera que, si bien dicho modelo impulsó importantes transformaciones, especialmente en el ámbito legal, aún persisten miedos, prejuicios y actitudes subyascentes, siendo la ley inofensiva contra las formas institucionalizadas discursivas de discriminación.

A continuación se presentarán los principales argumentos que los CDS enfrentan al modelo social materialista: la crítica a la concepción binaria Discapacidad/Deficiencia, y sus derivados: el olvido del cuerpo y la conformación de una identidad política colectiva fuerte. Seguidamente se expondrán otras perspectivas críticas actuales que problematizan especialmente la concepción de deficiencia.

\section{Concepción binaria}

El modelo social sostiene una forma binaria de concebir a la discapacidad que establece una separación tajante entre las deficiencias, consideradas como una limitación funcional y la discapacidad propiamente dicha, producto de la interacción con el entorno social, que puede ilustrarse bajo el slogan "discapacitados por la sociedad, no por nuestros cuerpos" (Shakespeare \& Watson, 2002). La escisión entre lo fisiológico y lo social fue una operación 
política e ideológica que permitió la generación de un movimiento activista fuerte cuyos logros se constituyeron en beneficios y equiparación de oportunidades para el colectivo identificado como personas con discapacidad, especialmente en el ámbito anglosajón (Ferrerira, 2011). Sin embargo, esta concepción dicotómica tuvo diversas críticas desde los estudios culturales, feministas, poscoloniales, de género y las teorías posmodernas (Corker, 1998; Davis, 2006; Ferreira, 2011; Hughes \& Paterson, 2008; Meekosha, 2011; Shakespeare 2006; Shakespeare \& Watson 2002).

Un cuestionamiento fue realizado por Corker (1998) utilizando las herramientas de análisis posestructuralistas para deconstruir los binarismos que se identifican como excluyentes, en su carácter totalizador, universalista y jerárquico. Estos binarismos están presentes en la estructura del pensamiento occidental moderno influido por las dicotomías: cuerpo/alma, real/ideal, naturaleza/ cultura, etc., cuyo rastro se remonta a Platón. Partiendo de esas premisas, la autora cuestiona la base biológica de la deficiencia y la concepción binaria del modelo social.

Siguiendo a Derrida (1968), utiliza el concepto de differance que incluye el doble sentido de diferenciar y diferir, para deconstruir las dicotomías: individual/social, deficiencia/discapacidad, normalidad/discapacidad, donde sostiene que el sentido permanece desplazado y aplazado, y que el significado incluye tanto lo que es como lo que no es. Así, para definirse, la normalidad, en el marco epistemológico occidental, necesitaría a la discapacidad y la discapacidad, desde las concepciones del modelo social, a la deficiencia. En este sentido, el modelo social de discapacidad propone una separación insostenible entre cuerpo y cultura, entre impedimento/deficiencia y discapacidad, sustentada en la construcción de un sujeto "cartesianizado" y escindido.

El modelo social de la discapacidad -pese a su crítica del modelo médico- entrega el cuerpo a la medicina y entiende al impedimento en los términos del discurso médico. Para recuperar ese espacio corpóreo perdido (...) el modelo social exige que se haga una crítica de su propia herencia dualista y que se establezca, en cuanto necesidad epistemológica, que el cuerpo con impedimentos es parte del dominio de la historia, 
cultura y el significado y no -como diría la medicina- un objeto no histórico, presocial y puramente natural. (Hughes \& Paterson, 2008, p. 108)

\section{La construcción de la corporalidad y el olvido del cuerpo}

Desde las teorías feministas se inicia desde la década del noventa una revisión teórica que problematiza la concepción de cuerpo y con ella la de deficiencia. Estos planteos denuncian las limitaciones tanto del modelo social como del modelo médico para dar cuenta de la complejidad del cuerpo como corporalidad, un cuerpo que no se reduce a los aspectos netamente biológicos y fisiológicos. "Así como las superficies corporales se presentan como lo natural, estas superficies pueden convertirse en el sitio de una actuación disonante y desnaturalizada que descubre el carácter performativo de lo natural en sí" (Butler, 2007, p. 284).

Uno de los principales aportes en el campo es el de Judith Butler, quien concibe la identidad sexual como una construcción móvil y contingente, como el resultado, nunca acabado ni cristalizado, de una performance, ${ }^{4}$ que incide sobre el cuerpo. La autora, al considerar al género como performativo, propone que lo considerado una esencia interna, un rasgo natural, por el contrario, es algo que anticipamos y construimos a través de un conjunto sostenido de actos y postulados. Ello implica la necesidad de, entre otras cuestiones, trascender las categorías simples de la identidad.

Las personas con discapacidad han sido frecuentemente representadas como asexuales, sin género, fenómenos (Meekosha, 2004). Los primeros cuestionamientos en relación al descuido o minimización de lo corporal en el modelo social materialista son realizados por Jenny Morris, Rosemarie Garland-Thomson, Carol

\footnotetext{
${ }^{4}$ Butler ubica la "performatividad" entre el lenguaje y su soporte corporal; como algo tanto lingüístico como teatral: "el acto discursivo es a la vez algo ejecutado [performed] (y por tanto teatral, que se presenta ante un público, y sujeto a interpretación), y lingüístico, que provoca una serie de efectos mediante su relación implícita con las convenciones lingüísticas. (...) el discurso mismo es un acto corporal con consecuencias lingüísticas específicas. Así, el discurso no es exclusivo ni de la presentación corpórea ni del lenguaje, y su condición de palabra y obra es ciertamente ambigua." (Butler, 2007, p. 31)
} 
Thomas, Sally French y Liz Crow (Shakespeare \& Watson, 2002), teniendo una gran repercusión y desarrollo en la actualidad (Corker, 2008; García-Santesmases Fernández, 2015; Hughes y Paterson, 2008; Meekosha, 2004; Pie Balaguer, 2012), quienes discuten la importancia de la deficiencia en la experiencia personal vital de los sujetos. La acusación se dirige hacia la exclusión de las deficiencias en su abordaje teórico. "El error epistemológico fue abandonar el 'cuerpo' como objeto de atención fundamental, asumiendo, con ello, que eso, el cuerpo, no es más que un substrato material, neutro y aproblemático, de nuestra existencia social" (Ferreira, 2011, p. 4).

En esta dirección, desde una posición crítica realista, se resalta que la deficiencia y las limitaciones funcionales singulares no son indiferentes o neutrales, sino que constituyen parte importante en la experiencia diaria, de la corporalidad y de la historia de vida de cada sujeto por tanto, al ignorarlas, se estaría suprimiendo parte de la biografía. Por lo cual, se apunta a la recuperación de la experiencia de la persona con discapacidad trascendiendo su representación como sujeto cartesiano descorporizado.

Por su parte, Shakespeare y Watson (2002) plantean asimismo que el abandono del cuerpo por parte del Modelo Social Materialista también se acompañó de un abandono de las investigaciones médicas, cirugías y tratamientos de rehabilitación por su estrecha relación con el modelo médico con el que confrontaban. Los autores proponen que se puede priorizar la eliminación de barreras sociales mientras que se sostienen formas de prevención y tratamientos médicos siendo ambos objetivos no excluyentes. Se realza la idea de que la "discapacidad" es producida por ambas dimensiones, social y corporal, y que ambas constituyen dos aspectos de una singular experiencia, imposible de definir con precisión sus límites.

Además, se puntualiza que la total remoción de barreras sociales no conlleva a la eliminación de la discapacidad, tal como lo proponen algunos autores del Modelo Social Materialista. Diversos trabajos, tales como los de Corker (1998) sobre la experiencia 
de las personas Sordas ${ }^{5}$ y Sally French sobre las problemáticas visuales concluyen en que ello no sucede (Shakespeare \& Watson, 2002). En esta dirección se plantea que la eliminación de las barreras contextuales es necesaria pero no suficiente ya que "dejaría intacta las estructuras discursivas y de comportamiento de las relaciones sociales, junto con sus consecuencias en la capacidad de acción social" (Corker, 2008, p. 133-134).

\section{Identidad política: nosotros/los otros}

El modelo social, a la par que el enfoque de derechos civiles de Estados Unidos, impulsó la conformación de la identidad clara y distintiva: "personas con discapacidad" para referir a un colectivo unido por su padecimiento ante la opresión social. Este concepto, surge, como se mencionó, en oposición a la visión médica dominante de la discapacidad, fuertemente individualista.

La unión de las personas evaluadas profesionalmente como "personas con discapacidad", conformando un único y gran grupo, permitió aunar esfuerzos y llevar adelante demandas y acciones colectivas que representaron un gran avance en la calidad de vida, en la recuperación de sus derechos e incluso de su dignidad.

Esta identidad ha sido importante en el ámbito del derecho y en la práctica política. Sin embargo, al decir de Shakesperare (2006) su fuerza también es su debilidad y esta adhesión a una identidad política común y fija, tal como plantea este modelo, ha recibido diferentes cuestionamientos.

En primer lugar, desde las actuales teorías sobre la identidad (Arfuch, 2005; Bauman, 2005) se problematiza su concepción estática y definitiva hacia una comprensión más fluida, multidimensional y contextual. Según Shakespeare y Watson (2002) algunas personas con deficiencias se resisten a la identificación como "discapacitados" porque ellos se ven a sí mismos como "normales". A la vez, otros, pueden asumir una identidad de una parte de su

${ }^{5}$ La Federación Mundial de Sordos (WFD) ha dictado como norma en el mundo el uso de la mayúscula cuando se hace referencia a las personas Sordas como integrantes de un grupo cultural, de una minoría lingüística. La comunidad Sorda argentina adhiere a esta propuesta. 
experiencia, por ejemplo, una mujer con discapacidad podría posicionarse más fuertemente como mujer, que como persona con discapacidad.

Entonces, resulta de interés el concepto de interseccionalidad que manifiesta la interacción de diversas estructuras de categorías que atraviesan a los sujetos: etnia, género, sexo, capacidad, entre otras, consideradas como "variables dependientes", cuestionando la restricción discursiva, la linealidad y monodisciplinariedad que caracterizan a los abordajes antecedentes. Un importante enfoque interseccional entre capacidad y heterosexualidad es la "Teoría de Crip” elaborada por Mc Ruer. La palabra Crip, deriva del término inglés cripple, que significa tullido, una palabra peyorativa, asociada al estigma y la burla, que muchas personas reivindican y hacen suya como una forma de desafío (Moscoso Pérez \& Arnau Ripollés, 2016). Ofrece un modelo cultural de la discapacidad, opuesto tanto al modelo médico como al modelo social. La teoría persigue examinar cómo los cuerpos y las mentes no normativas están oprimidas en un sistema de "compulsory able-bodiedness" o integridad corporal obligatoria, donde se concibe que no tener una discapacidad es el estado natural del deber ser, un estado altamente deseado.

En segundo lugar, se critica su exclusividad; dado que la inclusión en una identidad política común y la pertenencia a la colectividad de personas con discapacidad implica, como contracara, la exclusión tanto de otras identidades como de quienes no poseen los mismos atributos. Inclusión y exclusión son dos procesos complementarios que operan en simultaneidad, por lo tanto, asumir una identidad fuerte que afirma al colectivo "personas con discapacidad" refuerza la separación nosotros-los otros/ellos así como también las estructuras opresivas sociales (Meekosha \& Shuttleworth, 2009). Además, en la práctica, en la demarcación de esas categorías y determinación de cuáles sujetos pertenecen a cada grupo, continúa siendo necesario el criterio del profesional experto. Es decir, mantiene vigente, al modelo médico (poder médico, psicológico, psicopedagógico), para la medida de las deficiencias y la clasificación y certificación de las discapacidades. 
Desde una perspectiva opuesta, se propone el borramiento de la demarcación nosotros-los otros/ellos concibiendo que la deficiencia es ubicua siendo que no existe el cuerpo perfecto, sino que todos los sujetos experimentan o experimentarán limitaciones corporales. Desde esta perspectiva, lo "normal", lo común, lo más frecuente sería la "deficiencia."

En esta dirección, Davis (2006), en el marco de los CDS propone el concepto de dismodernidad para referirse a una era donde la "diferencia", lo "no-estándar" es lo que todos tenemos en común, la identidad no es fija sino móvil y la tecnología no está separada de nuestro cuerpo, sino que nos completa como también lo hace el otro, siendo la dependencia e interdependencia "la regla" y no la excepción. Según el autor la experiencia de las limitaciones del cuerpo es algo universal, aunque paradójicamente propio y singular. Así, propone una nueva ética sobre el cuerpo y resignifica los binarismos: deficiencia-normalidad, donde el primer término es la norma y el segundo la fantasía y el de dependencia-independencia, donde opone realidad con un pensamiento imposible.

\section{El canon de la normalidad}

Otra importante crítica problematiza el punto de enunciación de los discursos hegemónicos, develando relaciones de dominación y poder en las construcciones discursivas sobre determinados sujetos. En este marco, se denuncia la construcción tanto de la normalidad como de la anormalidad deficiente y, por tanto, de la deficiencia y del déficit, siendo que son conceptos indisociables y "no hay idea de déficit sin idea de normalidad" (Angelino, 2009, p.135).

En esta dirección, se visibiliza que la normalidad y sus normativas son producto de una larga historia de invenciones, situadas en determinados contextos, atravesadas por necesidades políticas, económicas y sociales. La construcción social, cultural, científica y política de la normalidad alcanzó su punto cumbre con el proyecto moderno y su ideario racional-racionalizador de producción de sujetos-individuos ciudadanos (Ferreira, 2011). La cristalización y 
fijación en la cultura occidental se produce especialmente sobre mediados del siglo XIX con la influencia de la nueva organización industrial, el pensamiento positivista y la teoría evolucionista desarrollada por Charles Darwin. Es así que el uso de la palabra normal para referir a la tipología común, estándar, regular y usual; como construcción, conformación de lo no desviante o diferente aparece en la lengua inglesa hacia 1840. La palabra "norma" en referencia a orden aparece en 1885, "normalidad" en 1849 y "normalización" en 1857 (Davis, 1997, p.10). Es posible decir, por lo tanto, que el par binario normalidad-anormalidad es un producto moderno (Foucault, 2000; Skliar, 2002).

A la vez, también en el siglo XIX, la sociología se alinea con la estadística en su pretensión de objetivación de los hechos sociales para conocerlos y, además, predecirlos. Así, el nuevo ciudadano se identifica con el "hombre medio", construcción teórica inexistente en la realidad, resultante de una fórmula matemática desarrollada por Adolphe Quetelet a mediados del siglo XIX (Quetelet, 1999). Se propone la existencia de un tipo humano cuyas variaciones están sujetas a la misma ley simétrica en su distribución. Esta ley de distribución se conoce como "Campana de Gauss" o "Bell Curve" y su formulación fue tomada de la curva de errores desarrollada por Gauss en el contexto de la astronomía. Esta representación ubica a un $95 \%$ de personas con determinadas características en el centro, que constituyen la mayoría y son identificadas como dentro de los umbrales de normalidad. Mientras que el $5 \%$ "restante" se encuentra por fuera de dichos parámetros. Así, los sujetos que se encuentran comprendidos dentro de este "resto" se identifican como "desvío", sea por exceso, superando las medidas del hombre medio, o por defecto, cuando "falta" algún atributo para alcanzar el Standard.

En esta dirección, las deficiencias se definen en términos de distancia con el hombre medio y se hace necesario medir a los sujetos con regla de la normalidad para identificar cuanto y como se aleja de los parámetros normales. Así será posible ubicarlo dentro de un sistema de clasificación y obrar acorde a sus singularidades. 


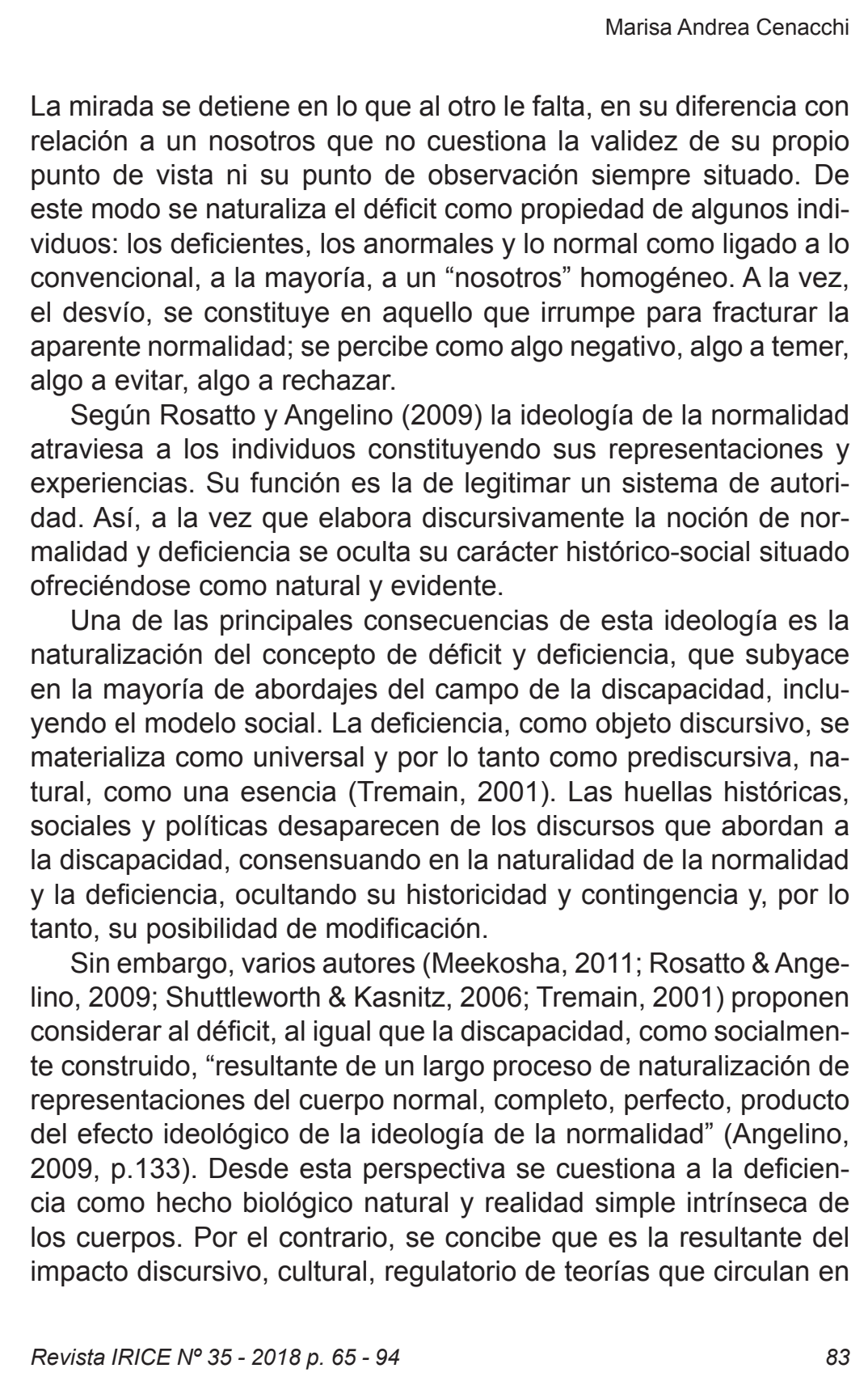


un determinado contexto situado, que modelan y moldean a los cuerpos.

Se considera entonces que "lo corporal es social (...). Lo social es, a su vez, corporal" (Ferreira, 2011, p. 4-5) en la medida en que estamos atravesados por discursos performativos sobre cómo debe ser nuestro cuerpo, como usarlo y por prácticas que producen tales efectos.

En este punto es posible observar que los contornos de la demarcación binaria deficiencia/discapacidad impulsada por el modelo social se diluyen y se funden al considerarse, por un lado, a la discapacidad necesariamente corporizada y por el otro a la deficiencia como construcción cultural, política y social.

\section{Críticas culturales y poscoloniales}

Algunos autores han estudiado a la Discapacidad con relación a su construcción como alteridad deficiente, a su designación social y cultural como otro, valiéndose de los planteos de los Estudios Culturales, Poscoloniales, Decoloniales y Subalternos y de las Epistemologías del Sur. Desde estas perspectivas se critica especialmente la representación de la discapacidad como una singularización transcultural, ahistórica, abstracta (Soldatic \& Grech, 2014), producto de suposiciones universales sobre las personas con discapacidad, concebidas como un colectivo homogéneo, culturalmente singular, que comparte intereses, perspectivas, objetivos y experiencias similares.

Desde un marco transcultural ("cross-cultural"), Shuttleworth y Kasnitz (2006) definen a la deficiencia como una percepción negativa culturalmente construida de una "anomalía" corporal, cognitiva o comportamental, y a la discapacidad como la respuesta social negativa a dicha percepción. En esta dirección analizan ejemplos de cómo diferentes grupos sociales construyen tanto las deficiencias como sus respuestas a ellas de manera singular y como la interseccionalidad impacta en esas construcciones, dependiendo de la situación contextual. 
Desde los Estudios Poscoloniales y Decoloniales ${ }^{6}$ se estudia y confronta al legado ideológico del imperialismo, con fuerte acento en el análisis discursivo y textual, resignificando en un formato posmoderno las exclusiones provocadas por las jerarquías epistémicas, espirituales, étnicas y de género desplegadas por la modernidad (Castro Gómez \& Grosfoguel, 2007).

Uno de los objetivos de la razón postcolonial (Mingolo, 2005) es el desmontaje de los discursos coloniales que construyen la identidad del otro y su representación a su imagen y "desemejanza”, como algo estático, fijo, homogéneo e inferior. La producción de conocimiento colonial está signada por la hegemonía del colonizador, como único habilitado para producir conocimientos válidos, fruto del eurocentrismo que funciona como locus epistémico desde el cual se erige el modelo del conocimiento, universalizando la experiencia local europea como modelo normativo (Grupo de Estudios sobre Colonialidad [GESCO], 2012).

Entones, bajo este marco, se construye la otredad desde su propia óptica situada, pero con pretensión de universalidad, configurando un saber que también se "trasplanta" al interior del colonizado, como si se tratara de su propio saber (Skliar, 2002) suprimiendo, además, la propia posibilidad enunciativa de los sujetos colonizados o subalternos (De la Vega, 2014).

El otro, cuya alteridad es irrepresentable, bajo la violencia epistémica (Palermo, 2010), es representado como una esencia unitaria, como una realidad que es posible conocer, clasificar y controlar; es fijado, cosificado y objetualizado.

Así, el otro colonial; anormal, deficiente, retardado, discapacitado, etc., es considerado separado del nosotros y representado desde un punto de vista externo, normativo, hegemónico, superior, completo y universal. Sus singularidades se expresan como falta, como déficit, como deficiencia, en relación con el estándar hegemónico

\footnotetext{
${ }^{6}$ Se concibe la descolonización como diferentes estrategias y formas contestatarias que plantean un cambio radical en las formas hegemónicas actuales de poder, ser, y conocer. Algunos investigadores son: Enrique Dussel, Aníbal Quijano, Walter Mignolo, Santiago Castro-Gómez, Zulma Palermo, Nelson Maldonado-Torres, entre otros.
} 
de normalidad. Un ejemplo claro es la invención oyente de la sordera y las prácticas colonialistas (Sherry, 2008; Skliar, 2000) sobre las personas Sordas, su lengua y sus tradiciones; prácticas que los obligan tanto a hablar como a pensarse, narrarse y juzgarse desde los parámetros oyentes, desde el discurso del déficit. "Son en estas prácticas donde muchos sordos se ven a sí mismos como deficientes e incompletos" (Skliar, 2000, p.8).

Según Skliar (2002) el otro colonial es temido por su otredad, su alteridad, que atenta contra la hegemonía del centro, su orden, su tranquilidad y pretensión de homogeneidad. En consecuencia, se propone una segunda forma de representar al otro: el "otro multicultural", que abandona su exotismo y exterioridad para estar junto a nosotros, siempre que sea distinguido, identificado y señalado claramente como otro. El otro es ahora tolerado, sin embargo ello implica el reconocimiento de las diferencias a la vez que la admisión de que las mismas son censurables. La tolerancia acontece en una relación asimétrica, donde el sujeto que tolera se ubica en una posición de superioridad sobre el sujeto que es tolerado; "no incluye la aceptación del valor del otro; por el contrario, es una (...) forma de reafirmar la inferioridad del otro y sirve de antesala a la intención de acabar con su especificidad" (Bauman, 1996, p. 82).

\section{Estudios Críticos Globales en Discapacidad}

Otro campo emergente de análisis crítico sobre la producción de la "alteridad deficiente," que se nutre de los estudios decoloniales y de las llamadas Epistemologías del Sur, ${ }^{7}$ es el de los "Estudios Críticos Globales en Discapacidad"8 ("Critical Global Disability Studies", en adelante CGDS) (Goodley, 2013; Meekosha, 2011).

\footnotetext{
${ }^{7}$ Concepto propuesto por Sousa Santos que implica un diálogo horizontal con los conocimientos de los subalternos colonizados en una ecología de saberes. Algunos investigadores son: Enrique Russel, Immanuel Wallerstein, Milton Santos, Aníbal Quijano, Walter Mignolo, Boaventura de Sousa Santos, Ramón Grosfoguel, Nelson Maldonado-Torres, Rinajit Guha, Gayatri C. Spivak, Edward Said, y otros.

${ }^{8}$ La línea de investigación posee una revista académica desde el año 2013: Disability and the Global South (DGS) disponible en Acceso Abierto https://dgsjournal. org/espanol/
} 
Sousa Santos (2011) define como Epistemologías del Sur a los nuevos procesos de producción de conocimientos a partir de las prácticas de grupos sociales que sufren desigualdades y discriminaciones. El autor, indica que el concepto de Sur Global no refiere a un espacio geográfico, aunque muchas veces coincida con él, sino a una metáfora del sufrimiento humano que también existiría en el Norte Global, en las minorías y grupos excluidos, marginados y silenciados.

Desde este posicionamiento de investigación en discapacidad, se cuestionan principalmente dos postulados; la responsabilidad del Norte Global en la producción de lo concebido como deficiencia y como discapacidad y la presencia de un enfoque eurocéntrico que subyace en las construcciones teóricas de los DS.

En relación al primer postulado, Paul Abberly (1987) fue el primero en proponer que la "deficiencia", concebida aquí desde su dimensión biológica, "es una producción social" (p.9), derivada mayormente del trabajo forzoso y/o desprotegido, los experimentos nucleares, las guerras, etc. Desde los CGDS se denuncia el colonialismo, imperialismo, y las guerras ${ }^{9}$ que, impulsadas por el Norte Global continúan "discapacitando"10 a gran número de personas del Sur Global (Goodley, 2013; Meekosha, 2008, 2011; Soldatic \& Grech, 2014).

En relación al segundo postulado, los CGDS revisan las concepciones anglosajonas y metropolitanas de la teoría y práctica de los DS a la luz de las experiencias del Sur Global, denunciando su "colonialismo académico" y la pretensión de universalidad de sus experiencias situadas en el Norte Global. Así, se expone que el análisis transcultural de la discapacidad, realizado generalmente

\footnotetext{
${ }^{9}$ Los conflictos armados se consideran una causa importante de producción de discapacidad en el mundo. Se sabe que en EEUU uno de cada 5 soldados que fueron a Iraq y Afganistán regresaron con alguna discapacidad. No se conocen los números iraquíes, pero Mercy Corsp estima que corresponden a una cifra que oscila entre tres y cinco millones (Meekosha, 2011).

10 En este marco se concibe el verbo "discapacitar" como un proceso deshumanizante que incluye la destrucción física, emocional, económica y cultural de una vida.
} 
por antropólogos en las DC, se enmarca en conceptos, problemáticas, objetivos y metodologías muy relacionados con su carácter ciudadano y vinculados al sistema de producción capitalista, Entonces, la recuperación de derechos que proponen, por ejemplo, de vida independiente, de autonomía, etc., resulta irrelevante para quienes luchan por su subsistencia.

En esta dirección, varios autores (Meekosha, 2011; Grech \& Soldatic, 2015) cuestionan los supuestos implícitos en los DS visibilizando sus limitaciones en la transposición fuera del Norte Global.

Los GSCD apuntan a la recuperación de las experiencias de las personas con discapacidad en el contexto del Sur Global y sus problemáticas, como son los de Ghai (2002), en la India; en Australia Meekosha (2008), entre una larga serie de autores. Proponen, por su parte, un concepto de discapacidad fluido y variable según las culturas y contextos; incluso reconocen que deficiencia, enfermedad, discapacidad no pueden ser fácilmente separadas en muchas culturas, acordando la existencia de multiplicidad de formas de describir y comprender la discapacidad y la deficiencia (Meekosha, 2011).

\section{Reflexiones finales}

Llegado a este punto, se han presentado los diversos modelos paradigmáticos y formas de pensar a la discapacidad que, como se ha mencionado, coexisten de forma implícita o explícita en el universo simbólico cotidiano y constituyen el marco para la posibilidad de las diferentes prácticas, valoraciones, normativas, concepciones, vinculaciones y relaciones con la alteridad deficiente.

Si bien actualmente hay consenso en concebir a la discapacidad como una construcción social, las principales divergencias teóricas se observan en la conceptualización de las deficiencias.

En efecto, desde las perspectivas vigentes que sustentan las políticas internacionales de derechos, basadas en el modelo social, se sostiene una división teórica entre discapacidad y deficiencia, manteniendo implícito, en la conceptualización de la deficien- 
cia tanto postulados del modelo médico como los conceptos de déficit y su contracara normalidad, curva normal, hombre medio, expuestos en el presente recorrido.

En esta dirección se perpetua la escisión "nosotros/los otros" habilitando prácticas colonialistas y/o compensatorias asimétricas sobre los sujetos categorizados como deficientes y certificados como personas con discapacidad.

Por el contrario, desde diversas perspectivas teóricas, se propone suprimir esa división binaria a partir de la complejización de las identidades y la consideración de las experiencias y significaciones situadas, concibiendo también a la deficiencia como socialmente construida. Así, cobra relevancia tanto la experiencia sensible corporal como los discursos y regulaciones culturales que impactan sobre el cuerpo, siempre situados, abriendo un nuevo campo problemático que habilita una profunda reflexión para repensar las políticas de inclusión educativa y sus puestas en obra, para así poder avanzar hacia prácticas educativas no excluyentes.

\section{Referencias}

Abberly, P. (1987). The Concept of Oppression and the Development of a Social Theory of Disability. Disability, Handicap and Society, 2(1), 5-19.

Aguado Díaz, A. (1995). Historia de las deficiencias. Madrid: Escuela Libre Editorial, Fundación ONCE.

Angelino, M. A. (2009). Ideología e ideología de la normalidad. En A. Rosatto \& M. A. Angelino (Coords.), Discapacidad e ideología de la normalidad. Desnaturalizar el déficit (pp.133-154). Buenos Aires: NovEduc.

Arfuch, L. (2005). Problemáticas de la identidad. En L. Arfuch (Comp.), Identidades, sujetos y subjetividades (pp.21-43). Buenos Aires: Prometeo libros.

Ball, S. (Comp.) (1993). Foucault y la educación. Disciplinas y saber. Madrid: Morata.

Barton, L. (2009). Estudios sobre discapacidad y la búsqueda de la inclusividad. Observaciones. Revista de Educación, 349, 137-152.

Bauman, Z. (1996). Modernidad y ambivalencia. En J. Berain (Comp.), Las consecuencias perversas de la modernidad (pp. 73-119). Barcelona: Anthropos. 

Goodley, D. (2013). Dis/entangling critical disability studies. Disability \& Society, 28(5), 631-644.

Grech, S. \& Soldatic, K. (2015). Disability and colonialism: (dis) encounters and anxious intersectionalities. Social Identities, 21(1), 1-5.

Grupo de Estudios sobre Colonialidad [GESCO] (2012). Estudios decoloniales. Un panorama general. KULA. Antropólogos del Atlántico Sur, 6, 8-21.

Hughes, B. \& Paterson, K. (2008). El modelo social de la discapacidad y la desaparición del cuerpo. Hacia una sociología del impedimento. En L. Barton (Coord.), Superar las barreras de la discapacidad (pp. 107-123). Madrid: Morata.

Linton, S. (2006). Reassigning meaning. En L. Davis (Ed.), The Disability Studies Reader. Second Edition (pp-161-172). Nueva York: Routledge, Taylor \& Francis Group.

Meekosha, H. (2004). Gender and disability. Sage Encyclopedia of Disability written in 2004. Recuperado de https://disability-studies. leeds.ac.uk/wp-content/uploads/sites/40/library/meekosha-meekosha. pdf

Meekosha, H. (2008). Contextualizing disability: developing southern/global theory. Presentación en la 4th Biennial Disability Studies Conference at Lancaster University, Reino Unido.

Meekosha, H. (2011). Decolonising disability: Thinking and acting globaly. Disability \& Society, 26(6), 667-682.

Meekosha, H. \& Shuttleworth, R. (2009). What's so 'critical' about critical disability studies? Australian Journal of Human Rights, 15(1), 47-75.

Mingolo, W. (2005). La razón postcolonial. Herencias Coloniales y Teorías Poscoloniales. AdVersuS, 2(4). Recuperado de: http://www.adversus. org/indice/nro4/articulos/articulo_mingolo.htm

Moscoso Pérez, M. \& Arnau Ripollés, S. (2016). Lo Queer y lo Crip, como formas de re-apropiación de la dignidad disidente. Una conversación con Robert McRuer. DILEMATA, 8(20), 137-144.

Oliver, M. (1998). ¿Una sociología de la discapacidad o una sociología discapacitada? En L. Barton (Ed.), Discapacidad y sociedad (pp. 3458). Madrid: Morata, Fundación Paiadeia.

Palacios, A. (2008). El modelo social de discapacidad: orígenes, caracterización y plasmación en la Convención Internacional sobre los Derechos de las Personas con Discapacidad. Madrid: CERMI.

Palermo, Z. (2010). Una violencia invisible: la colonialidad del saber. Cuadernos FHyCS-UNJu, 38, 79-88.

Pie Balaguer, A. (Coord.) (2012). Deconstruyendo la dependencia. Propuestas para una vida independiente. Barcelona: Editorial UOC. 


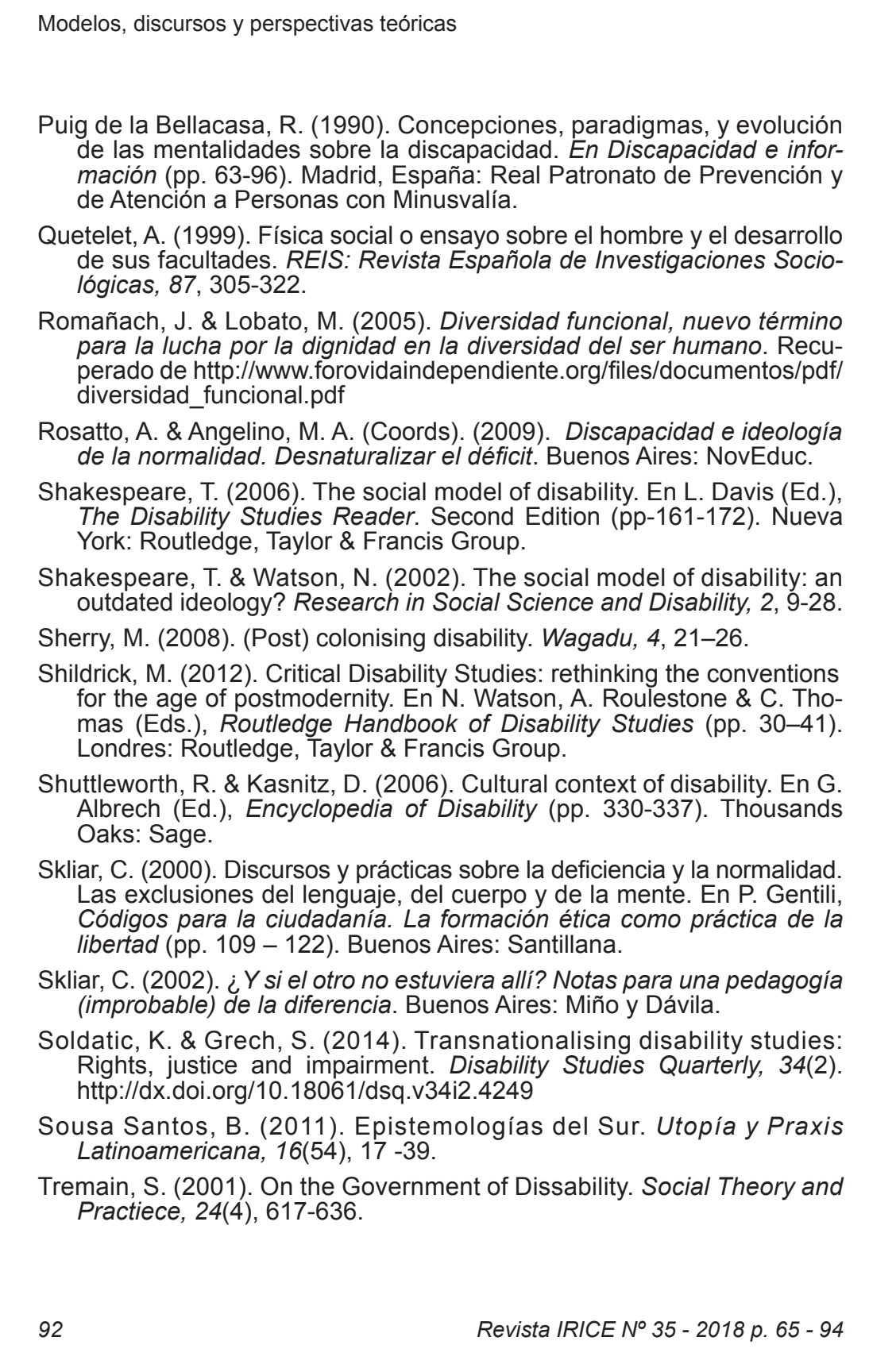


